\title{
PAPER \\ A Mapping Method for Multi-Process Execution on Dynamically Reconfigurable Processors
}

\author{
Vu MANH TUAN ${ }^{\dagger a)}$, Nonmember and Hideharu AMANO ${ }^{\dagger}$, Member
}

SUMMARY The multi-process execution in dynamically reconfigurable processors is a technique to enhance throughput by trying to exploit more inherent parallelism of applications. Basically, a total process for an application is divided into small processes, assigned into limited areas of a reconfigurable array, and concurrently executed in a pipelined manner. In order to improve the efficiency of the multi-process execution, a systematic method for mapping processes onto a reconfigurable array consisting of multiple hardware execution units is essential. This paper proposes and investigates a systematic method for mapping an application modeled as a Kahn Process Network onto a dynamically reconfigurable processing array. In order to execute streaming applications in a pipelined manner, the size of Tiles, which is a unit area of dynamically reconfigurable array, and the grouping of processes are adjusted. Using real applications such as DCT, JPEG encoder and Turbo encoder, the impact of different versions mapped onto the NEC Dynamically Reconfigurable Processor on performance is evaluated. Evaluation results show that our proposed mapping algorithm achieves the best performance in terms of the throughput and the execution time.

key words: dynamically reconfigurable processor, multi-process execution, single-process execution

\section{Introduction}

To date, a large number of researches in the area of reconfigurable computing have resulted in a number of academic and commercial Dynamically Reconfigurable Processing Arrays (DRPAs). These devices play an important role in balancing high performance demands and low power consumptions, especially in embedded devices. One of the trends in developing reconfigurable devices is the dynamic reconfigurability based on a multi-context mechanism such as DRP, DAPDNA-2, FE-GA and ADRES in order to minimize the reconfiguration overhead and greatly improve the performance of reconfigurable systems. The datapath mapped to a piece of physical hardware is called a context. A target application is divided into a set of different contexts, and a multi-context DRPA executes them by changing contexts with each clock cycle. Basically, with such multi-context DRPAs, an application is designed and mapped into hardware as a single thread of control. At any time, only one required context is activated and executed. In order to increase the throughput, some techniques such as software pipelining and loop unrolling can be applied to exploit more parallelism.

Manuscript received September 3, 2007.

Manuscript revised March 10, 2008.

${ }^{\dagger}$ The authors are with the Graduate School of Science and Technology, Keio University, Yokohama-shi, 223-8522 Japan.

a)E-mail: vmtuan@am.ics.keio.ac.jp

DOI: 10.1093/ietisy/e91-d.9.2312
For a particular hardware architecture, the performance improvement depends on the inherent parallelism of a target application. According to Hasegawa [1], the optimal size of processing element (PE) array in a DRPA is fixed related to the target application, and PEs exceeding the optimal number are not efficiently used. In many cases, the parallelism of an application is smaller than the number of PE array in a context. Accordingly, a large number of PEs is not used efficiently.

One of the methods to improve the performance in such a condition is making the best use of stream-level pipelined execution. Most target applications of such devices are stream processing, that is, data blocks to be processed are iteratively received in a certain interval. By dividing a total process into small independent sequential processes, and executing in a pipelined manner, a large number of PEs can be used efficiently.

Beside the popular single-process execution, where an application is designed and implemented as the only one thread of control for being assigned and executed on a reconfigurable array, some architectures like DRP-1 [2] by NEC Electronics support the "multi-process execution", which allows multiple threads of control to run concurrently. An application is divided into several processes; a large reconfigurable array is partitioned into some small arrays, each of which is called Tile; and each process is assigned to different Tiles of DRPAs, and executed in parallel. An interprocess communication mechanism using internal memories is defined for exchanging data between processes. Each Tile, to which a process is assigned, is independently controlled for executing and exchanging configuration data.

Although the introduction of the multi-process execution may lead to an effective way of partitioning applications in order to improve throughput and energy efficiency, systematic method for efficiently mapping processes into hardware execution units has not been well researched. This paper proposes a systematic mapping method to map target processes into Tiles, and shows examples applying it for DRP-1.

\section{Related Work}

There are a lot of research efforts aimed at models of computation such as Synchronous Dataflow (SDF) [3], Dataflow Process Networks [4], and Kahn Process Networks [5]. In the Ptolemy project[6], several models have been combined together to create a structure for the Ptolemy frame- 
work. However, this framework mainly focuses on application modeling and simulation without supporting the real mapping of application models onto models of architectures.

Partitioning applications is a well-known technique that has been thoroughly researched. HW/SW partitioning specifies which parts of an application should be mapped to hardware or software components [7]. Partitioning applications between reconfigurable hardware blocks of different granularity tries to map some parts of an application into fine-grain reconfigurable units and others into coarse-grain reconfigurable arrays for exploiting the advantages of various granularity reconfigurable modules [8]. In a multiprocessor environment, partitioning applications into multiple processes and threads, each of which could be mapped to an execution unit for running, is a well researched topic.

There are also a number of researches for job mapping and scheduling into the partitioned area of FieldProgrammable Gate Arrays (FPGAs) with partial reconfigurable capabilities. In these studies, the area and execution time requiring for a job are fixed, and scheduling algorithms decide the order and place where multiple jobs should be mapped. Since a three-dimensional (x, y, and temporal) placement problem must be solved for efficient placement, a number of theoretical researches have been done [9]-[11].

However, our target problem has the following differences, which prevent previous researches from being directly applied.

- In the multi-process execution treated here, each process being a part of a single job works in a pipelined manner, and handles a large number of streaming data arriving continuously. Thus, the throughput of a single job with multiple processes is a target for optimization.

- By a multi-context execution, a Tile can execute multiple processes sequentially as far as the number of contexts is sufficient.

- Multiple Tiles can be assigned to a single process for enhancing the execution speed by using parallel execution with a large number of PEs.

\section{Target Architecture and Application Model}

Figure 1 shows a general design flow. By explicitly specifying a mapping step, multiple target applications could be mapped onto a candidate architecture for evaluation. Typically, a set of applications is proposed for a certain architecture, then, they are mapped onto such an architecture. Through the performance analysis of each application-architecture-mapping combination, some improvements could be made for application implementation or mapping algorithm.

This paper will focus on a method for efficiently mapping an application modeled as a Kahn Process Network onto shown below target reconfigurable architectures.

\subsection{Target Architecture}

In this paper, a two-dimensional tile-based DRPA consisting of identical $M \times N$ hardware execution units is assumed to be our target architecture. This type of target architectures can be considered as a homogeneous structure, which allows more flexible in mapping.

As an example, a target DRPA $(4 \times 4)$ shown in Fig. 2 consists of a number of PE array units, each of which is called a Tile. A Tile has a certain size of PE array, distributed shared memory modules and its own controller for dynamic reconfiguration. The total number of Tiles in the target DRPA is referred as $N_{\text {all }}$. Here, a multi-context DRPA is assumed, that is, each Tile has a certain size of context memory and changes its hardware context according to a context pointer. The number of contexts that can be stored in the context memory $\left(C_{\max }\right)$ is limited in a certain number. Some examples of a candidate DRPA with correspondent parameters can be seen in Table 1, where PEs and Tiles show the total number of PEs and tiles in each DRPA respectively; PEs/tile denotes the number of PEs per a tile; and Contexts is the maximum number of contexts that can be stored in a PE.

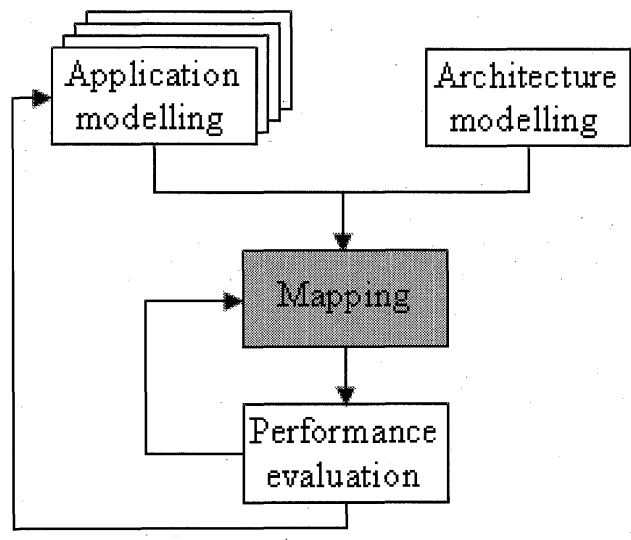

Fig. 1 General design flow.

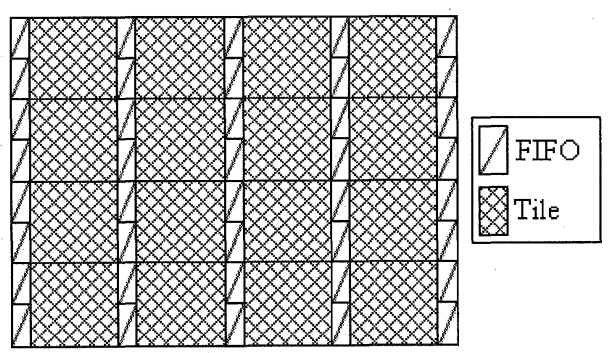

Fig. 2 Target DRPA.

Table 1 Example of possible target architectures.

\begin{tabular}{|l||c|c|c|}
\hline Parameter & DAPDNA-2 [12] & ADRES [13] & DRP-1 [2] \\
\hline PEs & 376 & 64 & 512 \\
\hline Tiles & 6 & 4 & 8 \\
\hline PEs/tile & 56 & 16 & 64 \\
\hline Contexts & 4 & 32 & 16 \\
\hline
\end{tabular}


Multiple neighboring Tiles can be joined to form a Tile group for executing a single task together. In this case, joined Tiles work synchronized with a single controller, and the number of available PEs becomes the sum of Tiles in the Tile group. Neighboring Tile groups can be communicated with each other through FIFOs formed with the distributed memory modules. That is, streaming data can be transferred between Tiles with a simple handshake mechanism.

\subsection{Target Application Model}

We assume that a target application can be represented with a Kahn Process Network (KPN), which is similar to a model proposed for streaming processors [14]. In this model, a total job is represented with multiple processes that can be executed in a pipelined manner. That is, data streams continuously arrive at a certain interval, and the results of a process are transferred to adjacent processes. A KPN has following characteristics:

- The execution of a KPN is deterministic, or in other words, for a given input, the same output is always produced. This model is suitable for stream-based multimedia and signal processing applications since it allows to model such types of applications, and guarantees that no data is lost.

- According to the model, processes are monotonic, that is, they require only a partial information from input for generating a partial result to output. Therefore, the model allows parallelism and pipelined processing, which are suitable to be implemented on reconfigurable arrays.

- The model makes it easier to partition an application into a set of parallel communicating processes.

For example, The upper part of Fig. 3 shows the KPN graph of a JPEG encoder. In this case, the graph becomes a linear structure. Media processing programs could be easily translated into KPNs [14], and here, we assume that the KPN corresponding to a target application has been already formed.

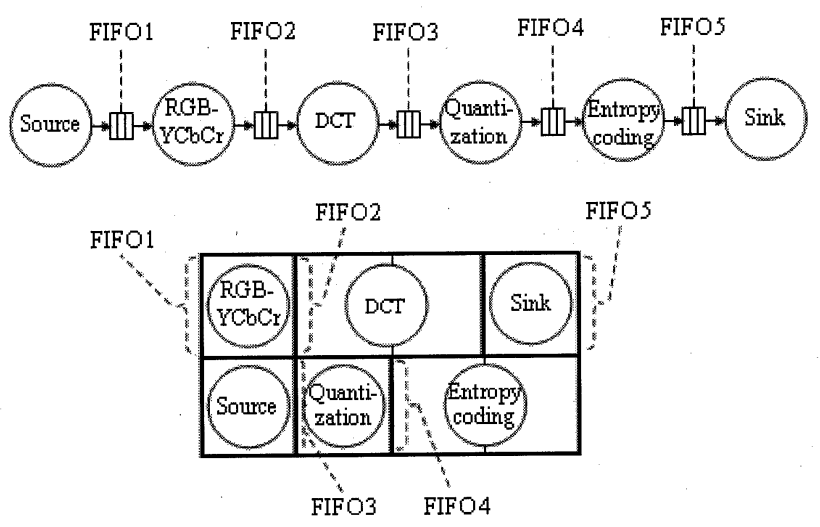

Fig. 3 Process mapping for JPEG encoder.

\subsection{Goal of Mapping}

Each process of a target KPN $\left(p_{i}\right)$ can be mapped into a Tile Group $T G_{j}$ of the target DRPA, and executed in a pipelined manner. The lower part of Fig. 3 shows an example of mapping for a JPEG encoder, in which, processes mapped into each Tile Group get data stream from the input FIFO, execute their own computation, and produce results to the output FIFO. If there is no data in the input FIFO or the output FIFO is full, the process execution is stalled. The data stream is assumed to arrive in a certain interval corresponding to the total throughput of the DRPA.

In order to improve the total throughput and the execution time of an application, it is critical to balance computation stages in the interrelation with other processes in a pipelined chain. In a pipelined processing model, the total throughput is bottlenecked with the most time consuming process. Here, by increasing the size of $T G_{j} \mathrm{~s}$, the throughput can be enhanced by parallel processing with more number of processing elements. If, for example, the process $D C T$ is the bottleneck of the JPEG encoder in Fig. 3, the total throughput can be improved by mapping $D C T$ into a $T G$ with a large size because the number of execution clock cycles of the bottleneck process (DCT) could be reduced.

The goal of mapping is to find the best combination of processes and Tile Groups in order to improve the throughput and to reduce the execution time of each pipeline stage while preserving system limitations: (1) the total number of Tiles used in $T G_{j}$ must be smaller than or equal to the number of Tiles supported in the target DRPA, and (2) the sum of contexts required for processes mapped in a $T G$ must be smaller than or equal to the number of contexts supported in the target DRPA.

\section{Mapping Algorithm}

\subsection{Target Process Graphs}

In this research, target process graphs are limited in a simple unidirectional linear graph with a fork-join structure. As shown in Fig. 4, a process can send a data stream to multiple processes (fork) and the results are gathered in the next process (join). Each process is connected with a FIFO, and can work independently. Stream data arrive in a certain interval to the starting process, and the total processes can be executed in a pipelined manner. Although complicated graphs cannot be represented because of the above limitations, most graphs of streaming processing are rather simple and fall into this limitation.

Here, process number $p_{i}$ is assigned into each process from the starting process to the terminal one. Parallel execution processes (processes 2, 3, and 4 in Fig. 4) can be assigned in any order. 


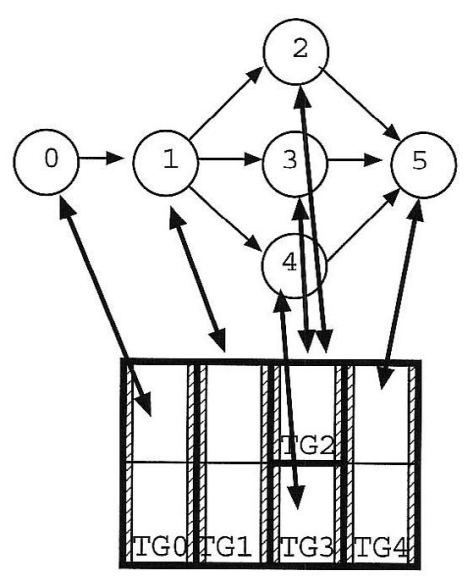

Fig. 4 The target process graph.

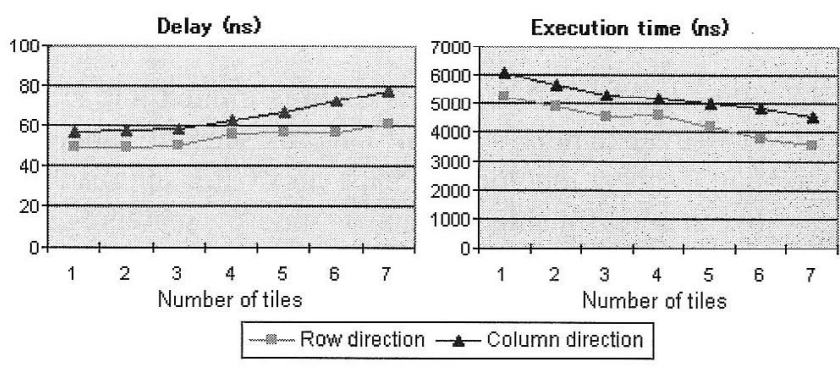

Fig. 5 Delay and execution time vs. number of tiles.

\subsection{Target Architecture and Process Mapping}

With the target architecture introduced in Sect. 3.1, neighboring Tiles can form a Tile Group $T G_{j}$, and neighboring $T G$ s can communicate via FIFOs provided on the edge of a Tile. The number of Tiles in a $T G_{j}$ is denoted with $\operatorname{Size}\left(T G_{j}\right)$.

When a process $p_{i}$ is mapped on a $T G_{j}$, different assignment strategies could affect the quality of an implementation. Namely, mapping depends on three following factors: 1) The number of tiles allocated to each process. 2) Absolute position of tiles. 3) And, absolute position of FIFOs for exchanging data between processes. Of which, the most important factor is the number of tiles assigned to a process. In general, the execution time represented with $F T\left(p_{i}, S i z e\left(T G_{j}\right)\right)$ is reduced when the process is executed with $T G$ formed by a large number of tiles. However, the relationship between the execution time and the number of tiles is not simple. For example, Fig. 5 shows the relationship between the number of execution clock cycles and the delay with various numbers of tiles when two main computation steps of two dimensional Discrete Cosine Transform (DCT), "Row direction" and "Column direction", are separately implemented on NEC Electronics DRP-1. While the number of execution clock cycles is decreased with a large number of tiles, the delay tends to be stretched. So, the total execution time is reduced but not relational to the size of $T G s$. Moreover, if a process does not have enough degree

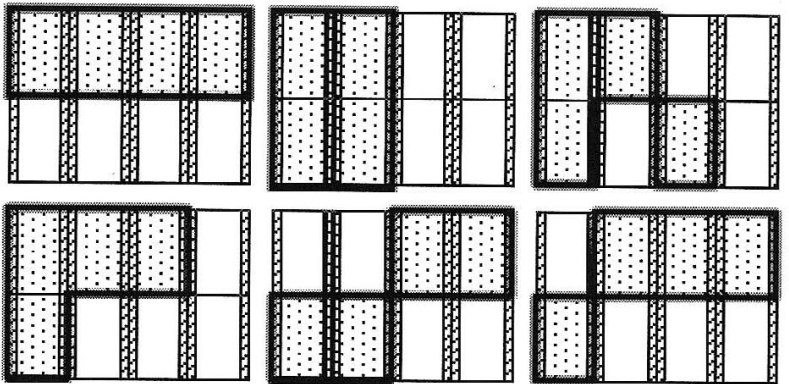

Fig. 6 Tile connection patterns for a $T G$.

of parallelism, the execution time is not improved at all by using tiles larger than a certain size.

Fortunately, by using design tools like Musketecr for DRP-1 [15], the relationship between the size of $T G s$ and the execution time can be evaluated in advance. Here, we assume that the execution time with the various size of $T G$ has been evaluated and the result represented with $F T\left(p_{i}\right.$, Size $\left.\left(T G_{j}\right)\right)$ can be obtained by table reference. Exactly speaking, $F T\left(p_{i}, \operatorname{Size}\left(T G_{j}\right)\right)$ depends not only on the size but also on the connected shape of Tiles. For example, when four Tiles are connected, the execution time slightly differs depending on the patterns shown in Fig. 6. However, implementation experience on DRP-1 shows that the difference is small when the number of connected Tiles are less than eight. So, we will ignore the difference here.

When multiple processes $p_{i}, \ldots, p_{i+k}$ are assigned into a $T G_{j}$, they are executed sequentially, (for example, process 2 and 3 mapped in TG2 in Fig. 4), that is, the total execution time $T_{i k}$ becomes $T_{i k}=\sum_{i}^{i+k} F T\left(p_{i}, \operatorname{Size}\left(T G_{j}\right)\right)(k>0)$.

Also, the number of contexts required for executing a process is depending on the number of Tiles in $T G_{j}$, and represented with $F C\left(p_{i}, S i z e\left(T G_{j}\right)\right)$. It can be also analyzed before scheduling by the design tools. When multiple processes are assigned into a $T G_{j}$, total required contexts becomes $C_{i k}=\sum_{i}^{i+k} F C\left(p_{i}, \operatorname{Size}\left(T G_{j}\right)\right)$.

Here, a process fork can be included in the graph (e.g. processes 2, 3 and 4 in Fig. 4). Such forked processes can be mapped into the same TG even if they have non-neighboring numbers (e.g. processes 2 and 4 in Fig. 4). However, if the graph does not have any fork, mapping processes with nonneighboring numbers will increase the communication between two processes, and this often requires the communication between non-neighboring Tiles. So, in order to make the algorithm simple, we only map the neighboring processes into a TG.

\subsection{Mapping Algorithm}

The proposed algorithm consists of three steps: process grouping, adjusting and topological mapping.

\subsubsection{Process Grouping}

Process grouping clusters the processes of an implementation in the specification model to $n$ groups in a way that the 
computation amount in each group is balanced. Neighboring numbered processes are mapped into a Tile as possible, keeping the limitation represented with $\sum_{i}^{i+k} F C\left(p_{i}, 1\right)<$ $C_{\max }$ for each Tile. Here, the number of mapped Tiles is called $N_{\text {used }}$ and so the number of unused Tiles is $N_{\text {all }}-N_{\text {used }}$. When there are multiple candidates for mapping, the one with less $N_{\text {used }}$ is selected. If $N_{\text {used }}>N_{\text {all }}$, the total job is too large to implement on the target DRPA. In this case, some of light-weight processes must be moved to the software executed on the embedded CPU with the DRPA. In the initial mapping, every mapped Tile Group $T G_{j}$ has only a Tile.

\subsubsection{Adjusting}

This step aims at adjusting the size of tile groups assigned in the previous step toward reducing the execution time of those taking a large time. First of all, the execution time of each $T G_{j}, \sum_{i}^{i+k} F T\left(p_{i}, \operatorname{Size}\left(T G_{j}\right)\right)$, is evaluated in order to find $T G_{j}$ s whose execution time is the largest. To improve the execution time of such $T G_{j}$, unused $n$ Tiles are assigned. Here, the limitation of the target DRPAs is considered. If the size of $T G$ must be 1 or even numbers (1, 2, 4, 6 and 8), the assigned number of Tiles must be the smallest one keeping the limitation.

There are three possibilities:

- All processes in $T G_{j}$ are executed sequentially with a larger size of Tile $\operatorname{Size}\left(T G_{j}\right)+n$. In this case, the execution time is $\sum_{i}^{i+k} F T\left(p_{i}, \operatorname{Size}\left(T G_{j}\right)+n\right)$. If the execution time is smaller than those of the other two possibilities, add $n$ Tiles to $T G_{j}$ to increase the size.

- The first $x$ processes $p_{i}, \ldots, p_{i+x-1}$ are executed with additional $n$ Tiles and the other processes are executed with $T G_{j}$. In this case, the execution time is $F T\left(p_{i}, n\right)+\cdots+F T\left(p_{i+x-1}, n\right)+F T\left(p_{i+x}+\operatorname{Size}\left(T G_{j}\right)\right)+$ $\cdots+F T\left(p_{i+k}, \operatorname{Size}\left(T G_{j}\right)\right)$. If the execution time is smaller than those of the other two possibilities, generate a new Tile Group and move $p_{i}, \ldots, p_{i+x-1}$ from $T G_{j}$.

- The last $x$ processes $p_{i+k-x}, \ldots, p_{i+k}$ are executed with separated $n$ Tiles and the other processes are executed with $T G_{j}$. In this case, the execution time is $F T\left(p_{i}, \operatorname{Size}\left(T G_{j}\right)\right)+\cdots+F T\left(p_{i+k-x}, \operatorname{Size}\left(T G_{j}\right)\right)+$ $F T\left(p_{i+k-x+1}, n\right)+\cdots+F T\left(p_{i+k}, n\right)$. If the execution time is smaller than those of the other two possibilities, generate new Tile Group and move $p_{i+k-x}, \ldots, p_{i+k}$ from $T G_{j}$.

After that, increase $N_{\text {used }}$ and iterate the above steps until all Tiles are used; that is $N_{\text {used }}$ is equal to $N_{\text {all }}$.

\subsubsection{Topological Mapping}

With the previous described steps, processes are mapped into $T G_{j}$, and the last step is to fit $T G_{j}$ into the physical shape of a $M \times N$ structure. Two approaches can be applied in this step.

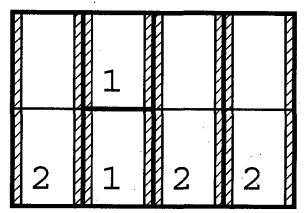

a)

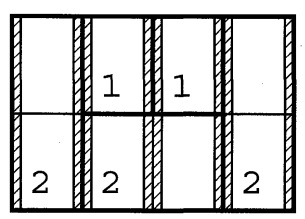

c)

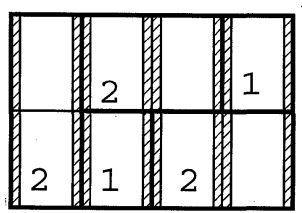

b)

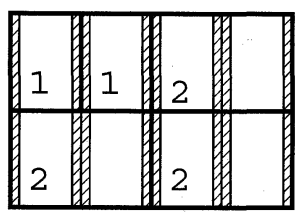

d)
Fig. 7 Example of tile assignment.

\section{(1) All possible mapping exploration}

Since the number of Tiles in a system is limited into small numbers (for example, eight in DRP as seen in Table 1), choosing the best topological mapping by this approach is possible in a reasonable amount of time by searching the complete solution space to retrieve all possible mapping variants.

In order to limit the search space, we decide the best allocation of Tiles for each list $\left(\operatorname{Size}\left(T G_{0}\right)\right.$, $\operatorname{Size}\left(T G_{1}\right), \ldots, \operatorname{Size}\left(T G_{k}\right)$ ), which consists of the sizes of TGs after adjusting where $k$ denotes the number of used TGs. There are multiple possibilities of Tile assignment for each list. For example, the allocations in Fig. 7 are all corresponding to the list $(2,1,1,2,2)$, since the arbitrary combination of tiles can be allowed in DRP-1. However, allocating a TG into separating tiles increases long wires which connect distant tiles, and degrades the operational frequency. Moreover, the communication between TGs is done through the FIFOs allocating edges of a tile, and so the neighboring TGs that need communication should be mapped into neighboring tiles. Considering them, we selected only one allocation for all possible patters in each list beforehand. For example, Fig. 7 (a) was selected for the combination $(2,1,1,2,2)$. It is called prepared pattern in the paper.

Figure 8 shows an example of the list $(2,1,1,4)$ and another example of $(2,1,1,2,2)$. In this method, for every branch of the list, a pattern is pre-assigned. Apparently, this method will cause the explosion of the possible patterns if the number of Tiles becomes large. This approach has been proved to be NP-complete, and it requires exponential time in order to find an optimal solution. However, for many upto-date DPRAs with a realistic size, the number of patterns are reasonable.

(2) Dynamic programming approach

In the possible solution space, the same sequence of physical tiles for a specific sequence of $T G_{j}$ often appears as a part of many mapping solutions. Instead of searching the whole solution space, the dynamic programming technique can be utilized to find the optimal topological mapping for 

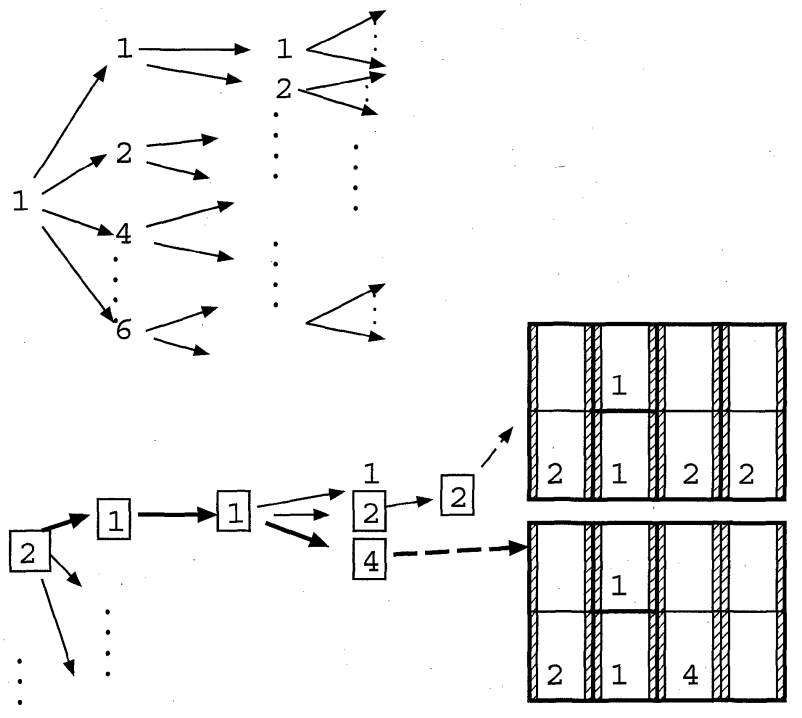

Fig. 8 Example of all possible mapping exploration approach.

the complete sequence of $T G_{j}$ by using solutions for smaller subsequences. Once an optimal solution for mapping up to $T G_{i}$ is determined, the execution time for executing up to $T G_{i+1}$ can be determined. This step is applied recursively to compute the final optimal mapping.

Given a sequence of Tile Groups $\left(\operatorname{Size}\left(T G_{0}\right)\right.$, Size $\left(T G_{1}\right), \ldots, S$ ize $\left(T G_{k}\right)$ ), the minimum execution time for executing up to process $i$ in a $T G_{j}, E_{i j}$ can be computed using the following recursive expression.

$$
E_{i j}=t_{i j}+\min \left(E_{i-1}\right)
$$

In the expression, $t_{i j}$ is the execution time of the process $i$ in the physical $T G_{j}$, and $\min \left(E_{i-1}\right)$ is the total of the minimum execution time of other processes up to process $(i-1)$. The expression shows that all possible ways of mapping process $i$ are examined once the mapping of process $(i-1)$ has completed.

\section{Target Device: DRP}

\subsection{DRP Architecture}

Here, for evaluation, we used a real DRPA named DRP1. It is a coarse-grain dynamically reconfigurable processor core released by NEC Electronics in 2002 [2]. It carries an on-chip configuration data corresponding to multiple contexts, and it dynamically reschedules them to realize multiple functions with one chip.

The primitive unit of DRP core is called a tile, and a DRP core consists of arbitrary number of tiles. The number of tiles can be expandable, horizontally and vertically. The primitive modules of a tile are processing elements (PEs), a State Transition Controller (STC), 2-ported Vertical Memories (VMEMs), and 1-ported Horizontal Memories (HMEMs). The structure of a tile is shown in Fig.9. There are 64 PEs located in one tile. The architecture of a

\begin{tabular}{|c|c|c|c|c|c|c|c|c|}
\hline \multicolumn{2}{|c|}{ HMEM } & \multicolumn{2}{|c|}{ HMEM } & \multicolumn{2}{|c|}{ HMEM } & \multicolumn{2}{|c|}{ HMEM } & \multirow{2}{*}{$\frac{\text { HMCTR }}{\text { VMEM }}$} \\
\hline PE & PE & PE & PE & PE & PE & PE & PE & \\
\hline PE & PE & PE & PE & $P E$ & $P E$ & PE & $\mathrm{PE}$ & VMEM \\
\hline PE & PE & PE & PE & PE & PE & PE & $P E$ & VMEM \\
\hline PE & PE & PE & PE & PE & PE & PE & PE & VMEM \\
\hline \multicolumn{8}{|c|}{ State Transition Controller } & VMCTR \\
\hline PE & PF & $P F$ & $P F$ & PF & Fم & $P F$ & Fסי & YMCM \\
\hline & & & & & & & & \\
\hline PE & PE & PE & PE & PE & PE & PE & PE & VMEM \\
\hline PE & PE & PE & PE & PE & $\mathrm{PE}$ & PE & PE & VMEM \\
\hline PE & $P E$ & PE & PE & $P E$ & $P E$ & $P E$ & $P E$ & VMEM \\
\hline
\end{tabular}

Fig. 9 DRP tile architecture.

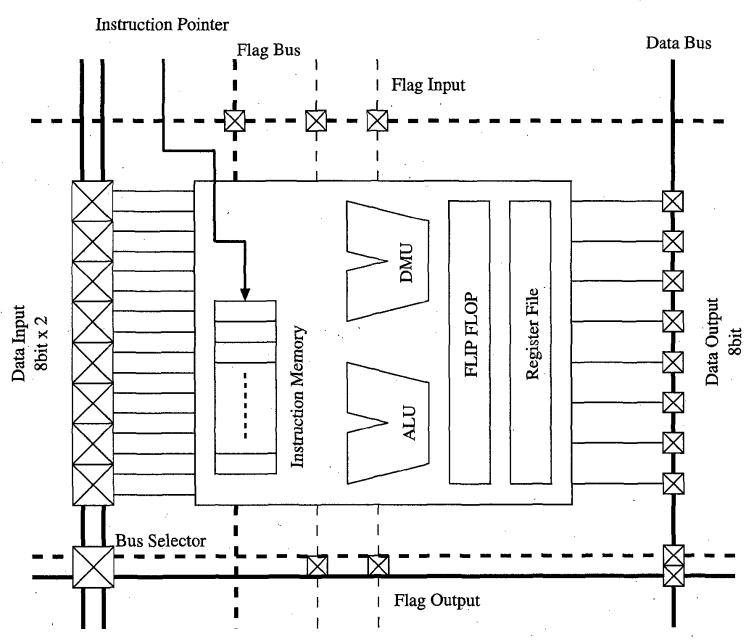

Fig. 10 Processing element architecture.

$\mathrm{PE}$ is shown in Fig. 10. It has an 8-bit ALU, an 8-bit data manipulation unit, sixteen 8-bit register file units, and an 8bit flip-flop. The STC is a programmable sequencer with in which any finite state machine can be stored. STC can receive event signals from $\mathrm{PEs}$ to branch conditionally.

As shown in Fig. 11, the prototype chip DRP-1 consists of a DRP Core with eight tiles. It is fabricated with 0.15 $\mu \mathrm{m}$ 8-metal layer CMOS process. It consists of 8-Tile DRP Core, eight 32-bit multipliers, an external SRAM controller, a PCI interface, and 256-bit I/Os. The maximum operation frequency is $100-\mathrm{MHz}$.

An integrated design environment [15] is provided including a high level synthesis tool, a design mapper for DRP, simulators, and a layout/viewer tool. Applications can be written in a C-based high level hardware description language, synthesized, and mapped directly onto the DRP-1.

The multi-process execution in DRP-1 is almost the same as the model introduced in Sect. 3. A process is assigned manually to a basic execution unit called a Tile. Since DRP-1 contains eight tiles (Fig. 11), the maximum number of processes that can be concurrently executed is 


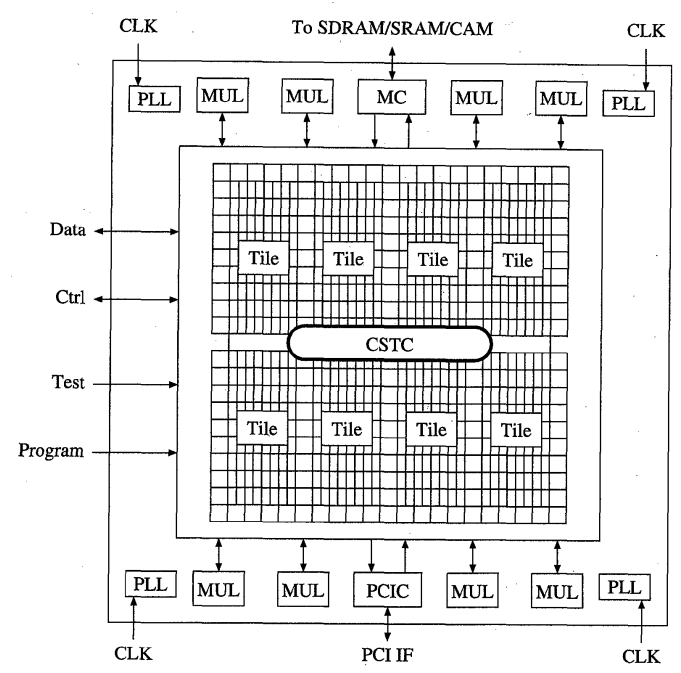

Fig. 11 Prototype chip: DRP-1.

eight. The DRP architecture is flexible enough to allow a process to be allocated to a group of continuous tiles, which provides a greater possibility for large processes to be implemented. STCs inside tiles can be operated and controlled independently to provide different instruction pointers for each process, so this allows processes to run in parallel. A first-in first-out (FIFO) memory mechanism, which employs VMEMs between tiles, is used as a inter-process communication method. A FIFO is for one-way communication and acts like a pipe. Writing to and reading from an FIFO are blocking, that is, a process needs to be stalled because of the data shortage.

\subsection{Mapping Applications onto DRP-1}

After an application is partitioned and modeled as a KPN, it is mapped into the 8-tile architecture of DRP-1 according to the mapping algorithm described in Sect. 4 with following constraints taking into account the specific features of DRP1.

- The limit number of tiles that can be allocated to an application no matter how many processes it is partitioned into is eight.

- Separate processes must be mapped into different tiles, or in other words, the scheduling of two or more processes sharing the same tile is forbidden in this implementation. This is because NEC's DRP-1, which is used as the target device for evaluation, does not support different processes to execute on the same tile sequentially. In order to solve this problem using the current design tool, we must manually prepare all possible combinations of merged processes in advance. It is not practical especially when the number of case studies becomes large. With devices providing a task controll mechanism (for example MuCCRA-1 or 2 [16]), this problem can be solved easily.

- A process can be mapped to a Tile Group formed in any shape (like the 4-tile example in Fig. 6).
An application is mapped onto the DRP architecture in a way that each process is mapped onto a tile or a Tile Group according to the mapping algorithm and each process FIFO is mapped one-to-one onto an DRP's FIFO. Moreover, processes that need to communicate are mapped to adjacent Tile Groups. An example mapping of the JPEG encoder is shown in Fig. 3.

\section{Evaluation}

\subsection{Target Applications}

Target applications listed in Table 2 have been implemented on DRP-1. Column "\#” denotes the number of processes each application is partitioned into. Column "Variant" shows different mapping versions for an application. Of which, the first version with the name of the application without number added is the one generated by the proposed mapping algorithm. Column "Mapping" shows the number of tiles each process in a KPN is mapped onto. For example, for the DCT, the column "Mapping" of the first line contains $1,3,3,1$. That means the DCT is modeled with four processes that are mapped to groups of 1, 3, 3, and 1 tile(s) respectively.

Each target application is partitioned into the processes of a KPN as follows.

- DCT: input, row-direction computation, columndirection computation, and output

- IMDCT: input, long-block computation, short-block calculation, and output

- Viterbi: input, trelis diagram, trace back, and output

- JPEG: input, RGB-YCbCr conversion, DCT, quantization, and entropy coding

- MPEG: input, variable length decode, inverse quantization, inverse discrete cosine transform, and motion compensation

- Turbo: input, permutation, interleave, encode, and multiplex

\subsection{Mapping Versions}

For comparison, the result of the single-process execution, where applications are not partitioned but the total process is mapped into 8 Tiles of the target architecture as only one thread of control, is provided in Table 2 with the line "Single" of column "Variant". Also, in this execution, column "\#" shows the value of 1 to clearly specify that applications are mapped as one process for executing.

For comparison, different mapping versions of applications shown in Table 2 with variety number of tiles assigned to processes are also evaluated. They are not the whole possible mapping space, but typical representatives. For demonstrating efficiency of the proposed algorithm with practical applications, there exist two problems: (1) there is no standard algorithm to be compared, and, (2) with simple algorithms (for example, random mapping), it is difficult 
Table 2 Implementation results of target applications.

\begin{tabular}{|c|c|c|c|c|c|c|c|c|c|}
\hline Application & Abbr. & \# & Variant & Mapping & $\begin{array}{c}\text { Critical path } \\
{[\mathrm{ns}]}\end{array}$ & $\begin{array}{l}\text { Clock } \\
\text { cycle }\end{array}$ & $\begin{array}{c}\text { Throughput } \\
\text { [Mbps] }\end{array}$ & $\begin{array}{c}\text { PEs/ } \\
\text { context }\end{array}$ & $\begin{array}{c}\text { Memories/ } \\
\text { context }\end{array}$ \\
\hline \multirow{7}{*}{ 2D DCT [17] } & \multirow{7}{*}{ DCT } & 1 & Single & & 67.3 & 95 & 160.2 & 98.1 & 11.0 \\
\hline & & \multirow{6}{*}{4} & $\overline{\mathrm{DCT}}$ & $1,3,3,1$ & $46: 8$ & 112 & 341.9 & 141.3 & 49.3 \\
\hline & & & DCT1 & $1,1,5,1$ & 63.7 & 182 & 143.5 & 142.5 & 48.2 \\
\hline & & & DCT2 & $1,1,3,1$ & 46.9 & 184 & 194.9 & 115.0 & 35.7 \\
\hline & & & DCT3 & $1,2,4,1$ & 59.5 & 136 & 195.6 & 139.1 & 46.7 \\
\hline & & & DCT4 & $1,2,2,1$ & 45.1 & 166 & 258.0 & 111.8 & 45.1 \\
\hline & & & DCT5 & $1,1,1,1$ & 44.3 & 238 & 180.6 & 93.7 & 26.4 \\
\hline \multirow{4}{*}{ IMDCT [17] } & \multirow{4}{*}{ IMDCT } & 1 & Single & & 140.6 & 1349 & 97.2 & 158.5 & 36.0 \\
\hline & & \multirow{3}{*}{4} & IMDCT & $1,3,3,1$ & 94.4 & 1452 & 182.3 & 185.9 & 39.0 \\
\hline & & & IMDCT1 & $1,4,2,1$ & 97.2 & 1421 & 178.6 & 188.0 & 40.1 \\
\hline & & & IMDCT2 & $1,2,2,1$ & 89.5 & 1592 & 169.8 & 178.3 & 39.0 \\
\hline \multirow{6}{*}{$\begin{array}{l}\text { Viterbi } \\
\text { decoder [17] }\end{array}$} & \multirow{6}{*}{ Viterbi } & 1 & Single & & 30.4 & 11 & 3.0 & 52.7 & 0 \\
\hline & & \multirow{5}{*}{4} & Viterbi & $1,2,2,1$ & 25.9 & 21 & 4.3 & 73.0 & 10.8 \\
\hline & & & Viterbi1 & $1,3,3,1$ & 33.5 & 18 & 3.7 & 84.0 & 10.8 \\
\hline & & & Viterbi2 & $1,4,2,1$ & 37.2 & 18 & 3.8 & 87.7 & 10.8 \\
\hline & & & Viterbi3 & $1,5,1,1$ & 48.1 & 17 & 3.5 & 85.2 & 10.8 \\
\hline & & & Viterbi4 & $1,1,1,1$ & 25.2 & 27 & 2.6 & 42.1 & 10.8 \\
\hline \multirow{4}{*}{$\begin{array}{l}\text { JPEG } \\
\text { encoder [18] }\end{array}$} & \multirow{4}{*}{ JPEG } & 1 & Single & & 56.3 & 636 & 42.9 & 136.3 & 19.8 \\
\hline & & \multirow{3}{*}{5} & JPEG & $1,2,2,1,2$ & 53.1 & 894 & 106.3 & 196.4 & 65.2 \\
\hline & & & JPEG1 & $1,1,2,1,2$ & 53.0 & 956 & 97.3 & 178.7 & 56.3 \\
\hline & & & JPEG2 & $1,1,3,1,2$ & 58.6 & 882 & 100.8 & 214.5 & 65.2 \\
\hline \multirow{5}{*}{$\begin{array}{l}\text { Turbo } \\
\text { encoder [20] }\end{array}$} & \multirow{5}{*}{ Turbo } & 1 & Single & & 81.3 & 16065 & 1.4 & 32.5 & 12.4 \\
\hline & & \multirow{4}{*}{5} & Turbo & $1,1,2,2,2$ & 58.3 & 17642 & 4.0 & 48.8 & 39.5 \\
\hline & & & Turbo1 & $1,1,1,3,2$ & 69.3 & 17498 & 3.9 & 54.2 & 39.5 \\
\hline & & & Turbo2 & $1,1,1,2,1$ & 58.1 & 18312 & 3.4 & 36.9 & 32.4 \\
\hline & & & Turbo3 & $1,1,2,2,1$ & 58.3 & 17936 & 3.9 & 31.1 & 39.5 \\
\hline \multirow{2}{*}{$\begin{array}{l}\text { MPEG-2 } \\
\text { decoder [19] }\end{array}$} & \multirow{2}{*}{ MPEG } & 1 & Single & & 73.9 & 1564 & 3.9 & 144.9 & 16.3 \\
\hline & & 5 & MPEG & $1,2,2,1,2$ & 62.9 & 1680 & 10.4 & 275.1 & 45.1 \\
\hline
\end{tabular}

to successfully complete the place-and-route phase and we cannot get the designs to evaluate their quality. In order to address this problem, we stretched the exploration space by relaxing the following conditions:

- Process grouping: Processes are grouped without taking into account balancing their computation amount. Other conditions are still kept to get the reasonable design.

- Adjusting: At this step, there are two options related to whether unused tiles are exploited to improve the execution time of $T G_{j}$. In a version, groups $T G_{j}$ are adjusted according to the proposed step in Sect. 4.3.2 with unused tiles in order to reduce the execution time of target groups. In the other version, groups $T G_{j}$ are not adjusted with unused tiles.

- Topological mapping: this step is performed exactly the same as proposed in Sect. 4.3.3.

These steps were performed almost automatically, and we tried to get as many results as possible. If the mapping results can successfully pass the place-and-route phase, it is recorded and assigned a tag number consisting the name of the application and a number. On the other hand, if the version fails at the place-and-route phase, it is not taken into consideration. For some applications, we could get a lot of mapping results, and Table 2 only shows results with good achievement either in the performance metrics: critical path, clock cycle and throughput. However, for example, in MPEG, we could not get any other mapping pattern than proposed method which passed the place-and-routing phase.

\subsection{Implementation Results}

Table 2 presents the performance result of target applications. In column "Variant", Single denotes the result of applications implemented in the single-process execution. Columns "PEs/context" and "Memories/context" show the average number of required PEs and used memories (both VMEMs and HMEMs) in a context.

In some applications like Viterbi, not all tiles are assigned because the parallelism of these applications is not high enough. Usually, the execution time could be reduced by increasing the number of tiles; but, for applications having low parallelism, only small number of PEs is required, so it is not expected to reduce execution clock cycles even with a larger number of tiles. Moreover, since using larger numbers of tiles with low usage will increase the leakage and the clock distribution power, an optimum number of tiles should be carefully selected for each implementation [1]. In many cases, this causes the actual number of tiles is not the largest one as shown in Table 2.

\subsection{Throughput}

The throughput of an implementation is represented with the amount of data processed in a second. Since all processes are executed in a pipelined manner in the multi-process execution, the throughput of the total execution is limited by 
the stage with the largest execution time.

Table 2 shows that all implementations in the multiprocess execution improve the throughput in a certain degree over the single-process execution up to nearly three time in JPEG implementation. In other words, by representing an application as a process network that are mapped into a DRPA as separate threads of control, the throughput could be improved. As the size of an input data block is the same, the throughput could be improved by either reducing the critical path or the number of execution clock cycles. In the multi-process execution, the critical path is usually shorter than that from the single-process execution. While a target application is mapped into the whole reconfigurable array in the single-process execution, in the multiprocess execution, the critical path of an application is the longest one among child processes, each of which is often mapped to one or several tiles but not the whole reconfigurable array. For example, in DCT, the implementation in the single-process execution requires 8 tiles of NEC's DRP, but in the multi-process execution, the largest process can only be mapped to 5 tiles (version DCT1 in Table 2).

Moreover, the calculation of the throughput in the multi-process execution takes into account the largest number of execution clock cycles among processes; and, by dividing an application into independent processes, that number is often smaller than that of a big process executing in the single-process execution though the total number of clock cycles from all processes is often larger due to process overhead. Since both the critical path and the number of execution clock cycles in the multi-process execution could be shorten, the throughput is likely to be improved.

The throughput could also be improved by taking advantages of the pipeline technique where multiple processes are arranged to operate in a pipelined manner. In most cases, the output of a computation step will be the input of the next step with no data or control hazard, so this is suitable for ${ }^{\circ}$ pipelining.

Among implementations in the multi-process execution mode, the one with our mapping algorithm achieves the best throughput. According to Hasegawa [1], each implementation has an optimum context size (the number of tiles) where the performance becomes optimum. Other context sizes no matter whether they are smaller or larger than the optimum one result in performance degradation. The implementation according to the mapping algorithm is likely the one where constituent processes are mapped with the optimum context sizes; therefore, the optimum throughput could be achieved. More importantly, in a pipelined environment, the throughput is greatly influenced by the balance of computation stages. The proposed mapping algorithm produces the most appropriate result in terms of process workload balancing. For example, balancing two processes, row-direction computation and column-direction computation, in DCT is the most important factor for the throughput since they occupy the largest part of the total execution time.

Although the proposed mapping algorithm could improve the throughput in a certain degree, the main limitation

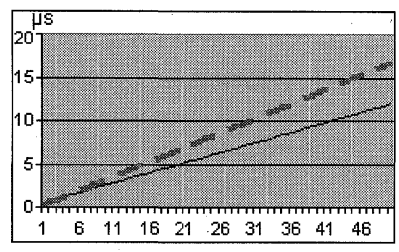

(a) Viterbi

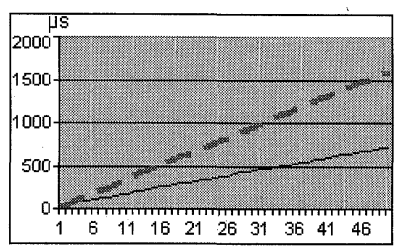

(b) JPEG

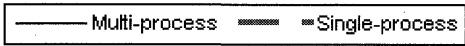

Fig. 12 Execution time vs. number of data blocks.

is the number of available contexts. Since the number of required contexts becomes easily more than 16 , the possibility of grouping processes are strictly limited. This is the reason why the execution time of each process is still unbalanced.

\subsection{Execution Time}

The execution time of an implementation for a given set of data can be computed as the product of the critical path and the number of execution clock cycles.

In the single-process execution, when multiple blocks of data are given, the number of execution clock cycles will be the multiple of that with one block of data and the number of data blocks. On the other hand, in the multi-process execution, because of the effect of pipelined processing, when more data are fetched, the number of execution clock cycles keeps increasing but less than the correspondence in the single-process execution. This is illustrated in Fig. 12, which shows the total execution time for Viterbi and JPEG when processing $N$ data blocks $(N=1,2, \ldots, n)$. In the graph, the largest number of data blocks $n$ equals to 50 . Other target applications show similar behavior. When a number of data block is small, the execution time in the single-process execution is smaller because of the overhead in the multi-process execution. However, when there are enough input data, or in other words, when a number of data block provided is larger than a certain number, to keep the pipeline stages full, the execution time in the multi-process execution becomes smaller.

From Table 2, among implementations in the multiprocess execution mode, the one with the proposed mapping algorithm achieves the smallest execution time since it has either the shortest critical path or the smallest execution clock cycle.

\subsection{Area Utilization}

In order to investigate the mapping quality, we evaluate the area utilization in terms of the number of active PEs and onchip memories. The overall area utilization could be presented by the number of consumed resources in every context. Since the number of contexts varies among applications, the average resource per a context will be used for examining the area utilization.

In Table 2, two columns "PEs/context" and "Memories/context" show the average number of active PEs and 
used memories in a context. They are calculated by dividing the total number of PEs and memories (both VMEMs and HMEMs) respectively for an implementation by the total number of contexts. From these two columns, it can be seen that, implementations in the multi-process execution demand more resources than correspondent implementations in the single-process execution. In terms of used PEs, the number of active PEs in the multi-process execution is often larger than that in the single-process execution. This is the result of having more than one process running at the same time.

Similarly, the requirement for active memories in the multi-process execution is also higher. One of the reasons is the use of FIFOs for inter-process communication. Even when requiring no memory for storing, an implementation still uses memories for communicating via FIFOs. This is illustrated in the implementation of a Viterbi decoder; while no memory for storing is demanded in the single-process execution, the multi-process execution still uses memories for FIFOs.

\subsection{Two Methods for Topological Mapping}

As mentioned in Sect. 4.3.3, there are two methods for topological mapping in the proposed algorithm: all possible mapping exploration and dynamic programming approach. The difference between these two methods is the time they take to produce the final result. In order to find the difference, a program for topological mapping according to both methods is implemented, then the execution time is computed. The programs are compiled by gcc 3.3.6 with -O3 optimization option and executed on a Pentium 4 processor $3.2 \mathrm{GHz}$ with $512 \mathrm{Mb}$ internal RAM.

Table 3 shows the time needed for mapping each target application onto the target application DRP-1. Columns "All" and "Dynamic" represents the methods of all possible mapping exploration and dynamic programming respectively.

Table 3 shows that the dynamic programming method greatly reduces time for topological mapping. Since target applications are not modeled with too many processes, and the target architecture only has eight tiles, the topological mapping step does not take too much time to produce the final result. Nonetheless, when the number of processes an application is modeled increases, or when the target architecture has more number of tiles, time for topological mapping will become a great concern. For example, taken time for applications modeled with four processes (DCT, IMDCT

Table 3 Time for topological mapping.

\begin{tabular}{|l||c|c|}
\hline Application & All (s) & Dynamic (s) \\
\hline DCT & 65.4 & 2.3 \\
\hline IMDCT & 65.3 & 2.3 \\
\hline Viterbi & 65.3 & 2.3 \\
\hline JPEG & 384.2 & 2.8 \\
\hline Turbo & 384.2 & 2.8 \\
\hline MPEG & 384.2 & 2.8 \\
\hline
\end{tabular}

and Viterbi) and applications modeled with five processes (JPEG, Turbo and MPEG) on the same target architecture is considerably different when the method of all possible mapping exploration is applied.

Table 3 also shows that time for topological mapping depends on the number of processes each application is divided into since applications with the same number of processes take almost the same time. Moreover, the number of tiles on the target DRPA influences time for mapping as well.

\section{Conclusion}

A systematic method for mapping an application modeled as a KPN onto a dynamically reconfigurable processing array is proposed. Using real applications and a real target architecture DRP-1, the impact of the proposed method on performance and area utilization is evaluated and analyzed. Evaluation results show that the throughput of the multiprocess execution increases from two to three times compared with the single-process execution, while more area utilization is realized as a result of processes being executed in parallel. In addition, our proposed mapping method results in the best throughput and execution time.

\section{Acknowledgments}

The authors sincerely express their gratitude to NEC Electronics and NEC laboratories.

\section{References}

[1] Y. Hasegawa, S. Abe, S. Kurotaki, V. Tuan, N. Katsura, T Nakamura, T. Nishimura, and H. Amano, "Performance and power analysis of time-multiplexed execution on dynamically reconfigurable processor," Proc. Reconfigurable Architecture Workshop, March 2006.

[2] M. Motomura, "A dynamically reconfigurable processor architecture," Microprocessor Forum, 2002.

[3] E.A. Lee and D.G. Messerschmitt, "Synchronous data flow," Proc. IEEE, vol.75, no.9, pp.1235-1245, Sept. 1987.

[4] E.A. Lee and T.M. Parks, "Dataflow process networks," Proc. IEEE, vol.83, no.5, pp.773-799, May 1995.

[5] G. Kahn, "The semantics of a simple language for parallel programming," Proc. IFIP Congress 74, North-Holland Publishing, 1974.

[6] J. Buck, S. Ha, E.A. Lee, and D.G. Messerschmitt, "Ptolemy: A framework for simulating and prototyping heterogeneous systems," Int. J. Computer Simulation, Aug. 1992.

[7] R. Niemann, Hardware/Software co-design for data flow dominated embedded systems, Kluwer Academic Püblishers, 1998.

[8] M.D. Galanis, A. Milidonis, G. Theodoridis, D. Soudris, and C.E. Goutis, "A partitioning methodology for accelerating applications in hybrid reconfigurable platforms," Proc. Design, Automation and Test in Europe, vol.3, pp.247-252, March 2005.

[9] K. Bazargan, R. Kastner, and M. Sarrafzadeh, "Fast template placement for reconfigurable computing systems," IEEE Des. Test Comput., vol.17, no.1, pp.68-83, March 2000.

[10] S.P. Fekete, E. Koehler, and J. Tech, "Optimal FPGA module placement with temporal precedence constraints," Proc. DATE, pp.658665, March 2001.

[11] P.-H. Yuh, C.-L. Yang, Y.-W. Chang, and H.-L. Chen, "Temporal floorplanning using 3D-subTCG," Proc. ASPDAC, pp.725-730, Jan. 
2004.

[12] T. Sugawara, K. Ide, and T. Sato, "Dynamically reconfigurable processor implemented with IPFlex's DAPDNA technology," IEICE Trans. Inf. \& Syst., vol.E87-D, no.8, pp.1997-2003, Aug. 2004.

[13] F.J. Veredas, M. Scheppler, W. Moffat, and B. Mei, "Custom implementation of the coarse-gained reconfigurable ADRES architecture for multimedia purposes," Proc. FPL, pp.106-111, Sept. 2005.

[14] B. Khailany, W.J. Dally, U.J. Kapasi, P. Mattson, J. Namkoong, J.D. Owens, B. Towles, A. Chang, and S. Rixner, "Imagine: Media processing with streams," IEEE Micro, vol.21, no.2, pp.35 46, March/April 2001.

[15] T. Toi, N. Nakamura, Y. Kato, T. Awashima, and K. Wakabayashi, "Iligh-level synthesis challenges and solutions for a dynamically reconfigurable processor," Proc. CICC, Nov. 2006.

[16] H. Amano, Y. Hasegawa, S. Tsutsumi, T. Nakamura, T. Nishimura, V. Tanbunheng, A. Parimala, T. Sano, and M. Kato, "MuCCRA chips: Configurable dynamically-reconfigurable processors," Proc. Solid-State Circuits Conference, ASSCC'07, pp.384-387, Nov, 2007.

[17] M. Suzuki, Y. Hasegawa, Y. Yamada, N. Kaneko, K. Deguchi, H. Amano, K. Anjo, M. Motomura, K. Wakabayashi, T. Toi, and T. Awashima, "Stream applications on the dynamically reconfigurable processor," Proc. International Conference on Field Programmable Technology, pp.137-144, Dec. 2004.

[18] Independent JPEG Group, http://www.ijg.org/

[19] MPEG Software Simulation Group (MSSG), http://www.mpeg.org/MPEG/MSSG

[20] C. Berrou and A. Glavieux, "Near optimum error correcting coding and decoding: Turbo codes," IEEE Trans. Commun., vol.44, no.10, pp.1261-1271, 1996.
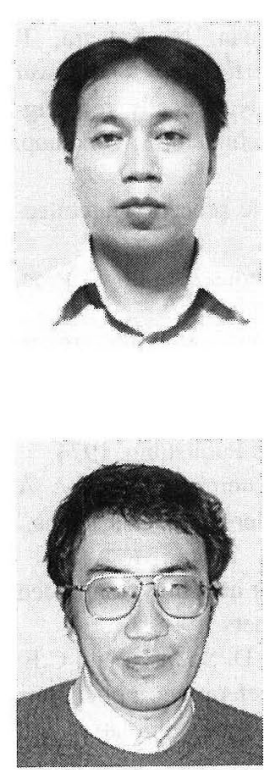

Vu Manh Tuan received the Bachelor degree at Hanoi University of Technology, Vietnam in 1993. In 2000, he received the Master of Science from University of New South Wales, Australia. He is now a second-year Ph.D student at AMANO Laboratory, Department of Information and Computer Science, Keio University. His research interests are reconfigurable architectures and algorithms.

Hideharu Amano received the Ph.D degree from Keio University, Japan in 1986. He is now a Professor in the Department of Information and Computer Science, Keio University. His research insterests include the area of parallel architectures and reconfigurable computing. 